\title{
GMR
}

\section{Changes in T-lymphocytes in lung cancer patients after hyperthermic intraperitoneal chemotherapy or radiotherapy}

\author{
L. Yan, M. Wu, N. Ba, G. Shi, L. Wang and H. Zhang \\ Department of Cancer Biotherapy Center, \\ The Fifth Affiliated Hospital of Zhengzhou University, Zhengzhou, China \\ Corresponding author: L. Yan \\ E-mail: yanlzheng@126.com \\ Genet. Mol. Res. 15 (2): gmr.15027865 \\ Received October 20, 2015 \\ Accepted January 15, 2016 \\ Published June 10, 2016 \\ DOI http://dx.doi.org/10.4238/gmr.15027865
}

\begin{abstract}
We investigated dynamic changes in T-lymphocyte subsets after hyperthermic intraperitoneal chemotherapy (HIPEC) or radiotherapy using flow cytometry. A total of 1423 lung cancer patients admitted to our hospital between October 2012 and July 2015 were enrolled, and age-matched healthy individuals served as controls. Peripheral blood mononuclear cells (PBMCs) were purified using standard Ficoll density gradient centrifugation, based on which $\mathrm{CD} 3+$, CD4+, and CD8+ T-cells were isolated. A surface marker was identified by flow cytometry. Immunohistochemical analysis determined the distribution of the cells in the tumor mass or adjacent tissues. A total of 957 patients (male: 555 ; female: 402 ; median age: 49.3 years) with lung cancer who had received only HIPEC or radiotherapy were enrolled. The patients were followed-up until death. No statistical difference was noticed between the patients who had received chemotherapy compared with the baseline levels. A remarkable elevation was noticed in the CD3+ T-cells in the patients three months after radiotherapy $(78.71 \pm$ 9.36 vs $68.15 \pm 9.65, \mathrm{P}<0.05)$. The level of $\mathrm{CD} 8+$ in the patients who had received chemotherapy or radiotherapy was remarkably elevated in
\end{abstract}


the post-treatment period $(\mathrm{P}<0.05)$. The $\mathrm{CD} 3+$ and $\mathrm{CD} 8+\mathrm{T}$-cells were mainly expressed in the cytoplasm rather than in the adjacent tissues. The expression of $\mathrm{CD} 3+$ and $\mathrm{CD} 4+$ was correlated to tumor infiltration and metastasis. Remarkable elevation was noticed in the CD3+ T-cells in the patients three months after radiotherapy. The expression of CD3+ and CD4+ was negatively correlated to tumor infiltration and metastasis in non-small-cell lung cancer patients.

Key words: Lung cancer; T-lymphocytes; CD3+; CD4+; CD8+

\section{INTRODUCTION}

Lung cancer, one of the major types of malignant cancer, is a great threat to public health worldwide (O'Rourke and Edwards, 2000; Paez et al., 2004). With treatment progression and scientific research, treatment plans including surgery, chemotherapy, radiotherapy, and other regimens have been developed; however, the incidence and mortality rate are still increasing. Unfortunately, most lung cancer patients are diagnosed at an advanced stage and limited treatment options are available. For these patients, chemotherapy and/or radiotherapy have been frequently used in clinical practice with the aim of controlling the progression or metastasis of cancer cells, or bringing the tumor lesions under control (Robinson et al., 2007; Scott et al., 2007; Azzoli et al., 2009).

Recently, extensive studies have been carried out on the efficiency of lung cancer treatments (Rosenberg et al., 2004; Grivennikov et al., 2010; Pardoll, 2012). Moreover, there are still some trials that aim to investigate the efficiency of immune therapy combined with chemotherapy or radiotherapy. As the disease in humans does not lend itself to randomized and controlled trials, it is difficult to accurately evaluate treatment efficiency. To the best of our knowledge, the function of the immune system is crucial for treatment. For example, evidence of lymphocytopenia has been used to predict a poor survival prognosis in advanced cancer patients (Lissoni et al., 2004). Nowadays, standard treatment efficiency evaluation methods have been proposed by the National Comprehensive Cancer Network (NCCN), which mainly rely on the evaluation of lesion size using the terms complete response, partial response, stable disease, and disease progression.

Because lung cancer is a progressive disease, it is necessary to evaluate the dynamic changes that occur in immune function based on which of the alternative treatment regimens are effective according to the patients' health. T-lymphocytes are recognized as the main effectors of antitumor immune responses (Phan et al., 2003). T-subsets have frequently been used in the evaluation of pathogenesis, treatment, and prognosis of many malignant tumors. For instance, CD3+ T-lymphocytes are the major lymphocyte subset in the peripheral circulation and also the main component of tumor-infiltrating lymphocytes (TILs) (Schaefer et al., 2005). In addition, CD4 T-cells play a critical role in mediating adaptive immunity to a variety of pathogens (Pasare and Medzhitov, 2003). They are also involved in autoimmunity, asthma, allergic responses, and tumor immunity. During T-cell receptor (TCR) activation in a particular cytokine milieu, naive CD4 T-cells may differentiate into one of several lineages of T-helper (Th) cells, including Th1, Th2, Th17, and iTreg. The presence of CD8+ T-cells in breast cancer is associated with a significant reduction in the relative risk of death from disease (Liyanage et al., 2002). However, such methods are mainly based on absolute counts 
or ratios with no dynamic observation. Therefore, in this study, we investigated the dynamic changes in T-lymphocyte subsets after hyperthermic intraperitoneal chemotherapy (HIPEC) or radiotherapy using flow cytometry.

\section{MATERIAL AND METHODS}

\section{Patients}

A total of 1423 lung cancer patients admitted to our hospital between October 2012 and July 2015 were enrolled in this study. The diagnosis of lung cancer was mainly based on guidelines proposed previously (Rivera et al., 2003). The clinical stage was categorized according to the Union for International Cancer Control (UICC) guidelines. Only patients who had received HIPEC or radiotherapy were included in this study. Patients with severe organ failure, osseous metastasis, or those who had received other therapies (e.g., target therapy or surgery) were excluded from the study. Written informed consent was obtained from each patient. The study protocols were approved by the Ethical Committee of the First Affiliated Hospital of Soochow University.

\section{Isolation of peripheral blood mononuclear cells (PBMCs)}

PBMCs were purified using standard Ficoll density gradient centrifugation according to the manufacturer instructions (Amersham Pharmacia, Uppsala, Sweden). CD3+ cells were sorted using a BD FACS Aria II cell sorting system (Becton Dickinson, CA, USA). CD4+ cells were isolated from PBMC using microbeads and an AutoMACS separation unit (Miltenyi Biotec) according to the manufacturer instructions. CD8+ cells were isolated using negative immunomagnetic separation techniques. The purity of the cells after separation was $>98 \%$.

\section{Flow cytometry}

After venous blood sample collection, flow cytometry was carried out using FACSCantoII (Becton Dickinson, NJ, USA). For the staining of cell-surface markers, 50 $\mu \mathrm{L}$ well-mixed, anticoagulated whole blood or $100 \mu \mathrm{L}$ leukocyte-activated whole blood was incubated with fluorochrome-conjugated monoclonal antibodies for $15 \mathrm{~min}$ in the dark at room temperature. With regards to the staining of intracellular cytokines, cell-surface staining was followed by fixation, permeabilization and intracellular staining. Fixation and permeabilization were performed using Fix \& Perm kits (Invitrogen Ltd., Paisley, Scotland) according to the manufacturer instructions. The antibodies used in this study were: FITC-CD3, FITC-CD4, and APC-CD8 (Becton Dickinson). Analyses were performed using the FACSDiva software (Becton Dickinson). Fluorescent-minus-one and isotype controls were used for gating.

\section{Immunohistochemical analysis}

The samples (tumor mass and adjacent tissues) were paraffin-embedded, and several heat-based antigen retrieval methods were used. After blocking of endogenous peroxidase activity by methanol containing $3 \%$ hydrogen peroxide, the tissue sections were incubated overnight at $4{ }^{\circ} \mathrm{C}$ with the primary antibodies, followed by the secondary antibodies. For 
the visualization of primary antibodies, we used the EnVision system (Dako). All tests were performed at least in triplicate.

\section{Follow-up}

The patients were followed-up until death. In each follow-up visit, the patients were required to undergo detection of $\mathrm{CD} 3+, \mathrm{CD} 4+$, and $\mathrm{CD} 8+$ cells.

\section{Statistical analysis}

The SPSS 17.0 software was used for the data analysis. Data are reported as means \pm standard deviation. Numerous data are presented as the percentage. The Student $t$-test was performed for intergroup comparison. The chi-square test was performed for numerous data analysis. A P value of $<0.05$ was considered statistically significant.

\section{RESULTS}

\section{Patient characteristics}

A total of 957 patients with lung cancer who had previously received only HIPEC or radiotherapy were enrolled in this study. There were 555 (57.99\%) male and $402(42.01 \%)$ female patients. The patient age ranged from 33.5 to 76.3 years (median, 49.3 years). Most of the patients $(\mathrm{N}=946)$ were at stages III or IV, but a few were at stage II $(\mathrm{N}=11)$ lung cancer. The patients were followed-up until death. Thirty age-matched healthy individuals were enrolled as controls. The patient characteristics are listed in Table 1.

Table 1. Patient characteristics.
\begin{tabular}{l|c|c|c|c}
\hline Variables & N & CD3+ & CD4+ & CD8+ \\
\hline Gender & & & $295(53.2 \%)$ & $310(55.9 \%)$ \\
\hline Male & 555 & $238(42.9 \%)$ & $206(51.2 \%)$ & $220(54.7 \%)$ \\
\hline Female & 402 & $178(44.3 \%)$ & $91(36.3 \%)$ & $100(39.9 \%)$ \\
\hline Differentiation & & & $212(58.9 \%)$ & $177(49.3 \%)$ \\
\hline Poorly & 251 & $108(43.0 \%)$ & $152(49.3 \%)$ & $160(46.2 \%)$ \\
\hline Moderately & 360 & $175(48.7 \%)$ & & \\
\hline Highly & 346 & $149(43.2 \%)$ & $5(45.5 \%)$ & $167(32.2 \%)$ \\
\hline Clinical stage & & & $240(43.6 \%)$ & $179(45.3 \%)$ \\
\hline Stage II & 11 & $4(36.4 \%)$ & & $222(40.3 \%)$ \\
\hline Stage III & 395 & $152(38.4 \%)$ & & \\
\hline Stage IV & 551 & $188(34.2 \%)$ & & \\
\hline
\end{tabular}

\section{Changes in CD3+, CD4+, and CD8+ T-cells in PBMCs}

The dynamic changes in $\mathrm{CD} 3+, \mathrm{CD} 4+$, and CD8+ T-cells in PBMCs are summarized in Table 2. The postoperative levels of the variables were obtained from the patients over a follow-up of more than 3 months. Compared with the baseline levels, no statistically significant difference was noticed in the patients who had received chemotherapy $(\mathrm{P}>0.05)$. However, a remarkable elevation was noticed in the CD3+ T-cells in the patients 3 months after radiotherapy $(78.71 \pm 9.36 v s 68.15 \pm 9.65, \mathrm{P}<0.05)$. For the expression of $\mathrm{CD} 4+$, no 
statistically significant difference was observed in the patients at each time duration. Compared with the baseline levels, the level of CD8+ in the patients who had received chemotherapy or radiotherapy was remarkably elevated in the post-treatment period $(\mathrm{P}<0.05)$.

Table 2. Dynamic changes in CD3+, CD4+, and CD8+ T-cells in peripheral blood mononuclear cells (PBMCs).

\begin{tabular}{l|c|c|c|c|c}
\hline \multirow{2}{*}{ Variables } & Pre-treatment level & \multicolumn{2}{|c|}{ Intra-treatment level } & \multicolumn{2}{c}{ Post-treatment level } \\
\cline { 3 - 6 } & & Chemotherapy & Radiotherapy & Chemotherapy & Radiotherapy \\
\hline CD3+ & $68.15 \pm 9.65$ & $68.95 \pm 9.84$ & $74.53 \pm 10.62$ & $72.39 \pm 9.81$ & $78.71 \pm 9.36$ \\
\hline CD4+ & $33.52 \pm 6.78$ & $35.69 \pm 6.98$ & $30.27 \pm 10.68$ & $29.34 \pm 8.76$ & $28.26 \pm 8.17$ \\
\hline CD8+ & $38.26 \pm 7.86$ & $39.65 \pm 9.62$ & $43.28 \pm 6.97$ & $44.29 \pm 9.26$ & $45.96 \pm 10.48$ \\
\hline
\end{tabular}

In this study, subgroup analysis was also performed, in which we compared the changes in $\mathrm{CD} 3+, \mathrm{CD} 4+$, and CD8+ T-cells in PBMCs in patients of various types, including non-small cell lung cancer (NSCLC) and small cell lung cancer (SCLC). The results revealed no statistically significant difference between the baseline levels of the NSCLC patients and those of the SCLC patients. Moreover, no statistically significant difference was noticed in the intra-treatment and post-treatment levels between these patients. These results indicate that radiotherapy caused no changes in the $\mathrm{CD} 3+, \mathrm{CD} 4+$, and $\mathrm{CD} 8+$ T-cells in PBMCs compared with the chemotherapy regimen. However, notable differences were observed in the expression of $\mathrm{CD} 3+$ and $\mathrm{CD} 8+$ in the post-treatment groups compared with the baseline levels.

\section{Expression of CD3+, CD4+, and CD8+ T-cells in the tumor mass and the adjacent tissues}

In this study, we also determined the expression of T-cells in the tumor mass and the matched adjacent tissues. CD3+ and CD8+ T-cells were mainly expressed in the cytoplasm with a positive rate of up to $65 \%$, which was superior to that of the adjacent tissues (Figure 1). However, CD4+ T-cells were observed in the tumor tissues and the adjacent tissues, with no statistically significant difference between them $(\mathrm{P}>0.05$, Figure 1$)$.

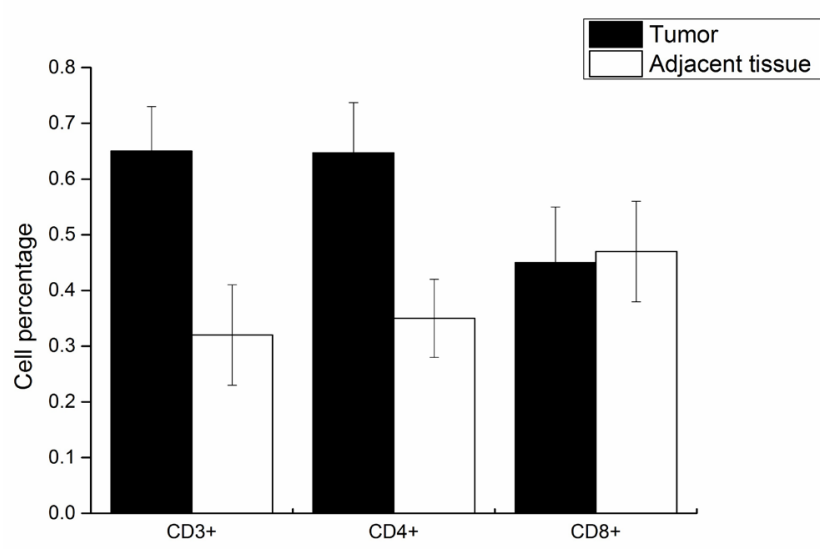

Figure 1. Proportion of $\mathrm{CD} 3+, \mathrm{CD} 4+$, and $\mathrm{CD} 8+$ cells in the tumor mass and the adjacent tissues. The tests were performed in triplicate at least. The data are reported as means \pm standard deviation. 


\section{Correlation between prognosis and the expression of $\mathrm{CD3}+, \mathrm{CD} 4+$, and $\mathrm{CD8}+$ T-cells in PMBCs}

The expression of $\mathrm{CD} 3+$ and $\mathrm{CD} 4+$ was correlated to tumor infiltration and metastasis. However, no correlation was noticed between CD8+ and tumor infiltration and metastasis. For the subgroup analysis, we also determined the expression of these markers in the NSCLC or SCLC patients (Table 3). No remarkable correlation was observed between the expression of CD3+, CD4+, and CD8+ T-cells in PMBCs in the SCLC patients. However, in the NSCLC patients, the expression of $\mathrm{CD} 3+$ and $\mathrm{CD} 4+$ was negatively correlated to tumor infiltration and metastasis.

\section{DISCUSSION}

Lung cancer refers to a malignant lung tumor characterized by uncontrolled cell growth in pulmonary tissues. Common treatment options include surgery, chemotherapy, radiotherapy, and target therapy. In this study, we investigated the dynamic changes in T-lymphocyte subsets after chemotherapy or radiotherapy using flow cytometry. In addition, we also examined the correlation between these T-cell subsets and infiltration and metastasis.

Currently, the diagnosis and staging of lung cancer is highly focused on the detection and anatomic distribution of the tumor (Hyer and Silvestri, 2000). Moreover, advanced efforts in molecular staging have paid more attention to the characteristics of the tumor including the tumor size. To date, immune therapy is considered an alternative method for the treatment of lung cancer. It has emerged in recent years as a promising therapeutic approach for treating lung cancer (Homey et al., 2002). Two approaches are of particular importance to immune therapy. One is through immune checkpoint inhibition, with the aim of counteracting the physiological mechanisms of immune tolerance co-opted by the tumor. The other is vaccine therapy, which enables enhanced exposure to tumor antigens. T-lymphocyte-mediated tumor antigen recognition is considered to be at the core of cancer immunotherapy. Additionally, T-lymphocytes have frequently been used in the evaluation of pathogenesis, treatment, and prognosis of many malignant tumors. As is well known, T-cell adoptive immunotherapy is highly reliant on abundant cells with satisfactory proliferative capacity and intact effector functions. In a previous study, an imbalance of surface receptors NKG2D and NKG2A in $\mathrm{CD} 3+, \mathrm{CD} 4+$, and $\mathrm{CD} 8+$ T-lymphocytes was reported to be associated with a poor prognosis and disease progression in patients with advanced cancers (Yu et al., 2014). Moreover, intra-tumor infiltration mediated by CD3 + cells is related to longer post-operative survival in patients with lung cancer (Schoo and Coppes, 1976). CD4+ T-cells in cancer stroma are associated with favorable prognoses in human NSCLC. Further, infiltration of CD8+ T-cells in NSCLC is related to the dedifferentiation of cancer cells rather than prognosis (Mori et al., 2000). In this study, we compared the CD3+, CD4+, and CD8+ cells in PBMCs in patients with lung cancer, as well as the tumor mass and the adjacent tissues. The results indicated no statistically significant difference in the patients who had received chemotherapy compared with the baseline levels $(\mathrm{P}>0.05)$. However, remarkable elevation was noticed in the CD3+ T-cells in the patients 3 months after radiotherapy. In addition, compared with the baseline levels, the level of CD8+ in the patients who had received chemotherapy or radiotherapy was remarkably elevated in the post-treatment period $(\mathrm{P}<0.05)$. 
T-lymphocyte imbalance after HIPEC or radiotherapy

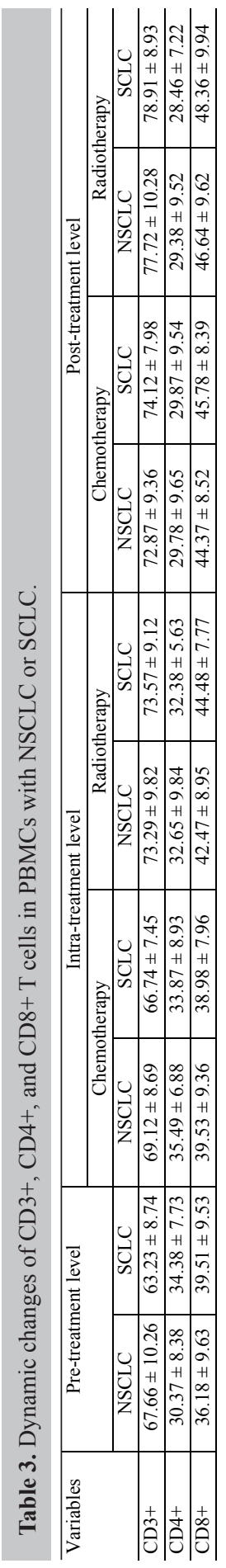

Genetics and Molecular Research 15 (2): gmr.15027865 
In this study, we also determined the expression of the T-cells in the tumor mass and the matched adjacent tissue. As with the previous study, the CD3+ and CD8+ T-cells were mainly expressed in the cytoplasm with a positive rate of up to $65 \%$; whereas CD4+ was observed in the tumor tissues and the adjacent tissues, with no statistically significant difference identified.

Extensive studies have been carried out to investigate the expression of T-cell subsets, and prognosis and treatment efficiency. For example, higher CD3+, CD4+, and CD8+ intratumoral and stromal counts are associated with a good prognosis (Rathore et al., 2014). In addition, low density of $\mathrm{CD} 3+, \mathrm{CD} 4+$, and $\mathrm{CD} 8+$ cells may increase the risk of relapse in squamous cell cervical cancer (Nedergaard et al., 2007). Further, higher numbers of CD3+, CD4+, and CD8+ tumor-infiltrating lymphocytes (TILs) is a predictor of extended survival in patients with infiltrating ductal carcinoma of the breast (Dushyanthen et al., 2015). In this study, the expression of CD3+ and CD4+ was correlated to tumor infiltration and metastasis. However, no correlation was noticed between CD8+ and tumor infiltration and metastasis. The subgroup analysis revealed no remarkable correlation between the expression of CD3+, CD4+, and CD8+ T-cells in PMBCs in the SCLC patients. However, in the NSCLC patients, the expression of $\mathrm{CD} 3+$ and $\mathrm{CD} 4+$ was negatively correlated to tumor infiltration and metastasis.

In conclusion, no statistically significant difference was observed in the patients who had received chemotherapy compared with the baseline levels. Remarkable elevation was noticed in the CD3+ T-cells in the patients 3 months after radiotherapy. The expression of CD3+ and CD4+ was negatively correlated to tumor infiltration and metastasis in NSCLC patients.

\section{Conflicts of interest}

The authors declare no conflict of interest.

\section{REFERENCES}

Azzoli CG, Baker S, Jr., Temin S, Pao W, et al.; American Society of Clinical Oncology (2009). American Society of Clinical Oncology Clinical Practice Guideline update on chemotherapy for stage IV non-small-cell lung cancer. $J$. Clin. Oncol. 27: 6251-6266. http://dx.doi.org/10.1200/JCO.2009.23.5622

Dushyanthen S, Savas P, Willard-Gallo K, Denkert C, et al. (2015). Tumour-infiltrating lymphocytes (TILs) in breast cancer: a predictive or a prognostic marker? Curr. Breast Cancer Rep. 7: 59-70. http://dx.doi.org/10.1007/s12609014-0178-4

Grivennikov SI, Greten FR and Karin M (2010). Immunity, inflammation, and cancer. Cell 140: 883-899. http://dx.doi. org/10.1016/j.cell.2010.01.025

Homey B, Müller A and Zlotnik A (2002). Chemokines: agents for the immunotherapy of cancer? Nat. Rev. Immunol. 2: 175-184. http://dx.doi.org/10.1038/nri748

Hyer JD and Silvestri G (2000). Diagnosis and staging of lung cancer. Clin. Chest Med. 21: 95-106, viii-ix. http://dx.doi. org/10.1016/S0272-5231(05)70010-1

Lissoni P, Brivio F, Fumagalli L, Messina G, et al. (2004). Efficacy of cancer chemotherapy in relation to the pretreatment number of lymphocytes in patients with metastatic solid tumors. Int. J. Biol. Markers 19: 135-140.

Liyanage UK, Moore TT, Joo HG, Tanaka Y, et al. (2002). Prevalence of regulatory T cells is increased in peripheral blood and tumor microenvironment of patients with pancreas or breast adenocarcinoma. J. Immunol. 169: 2756-2761. http://dx.doi.org/10.4049/jimmunol.169.5.2756

Mori M, Ohtani H, Naito Y, Sagawa M, et al. (2000). Infiltration of CD8+ T cells in non-small cell lung cancer is associated with dedifferentiation of cancer cells, but not with prognosis. Tohoku J. Exp. Med. 191: 113-118. http://dx.doi. org/10.1620/tjem.191.113

Nedergaard BS, Ladekarl M, Thomsen HF, Nyengaard JR, et al. (2007). Low density of CD3+, CD4+ and CD8+ cells is associated with increased risk of relapse in squamous cell cervical cancer. Br. J. Cancer 97: 1135-1138. http://dx.doi. org/10.1038/sj.bjc. 6604001 
O'Rourke N and Edwards R (2000). Lung cancer treatment waiting times and tumour growth. Clin. Oncol. (R Coll Radiol) 12: 141-144. http://dx.doi.org/10.1053/clon.2000.9139

Paez JG, Jänne PA, Lee JC, Tracy S, et al. (2004). EGFR mutations in lung cancer: correlation with clinical response to gefitinib therapy. Science 304: 1497-1500. http://dx.doi.org/10.1126/science.1099314

Pardoll DM (2012). The blockade of immune checkpoints in cancer immunotherapy. Nat. Rev. Cancer 12: 252-264. http:// dx.doi.org/10.1038/nrc3239

Pasare C and Medzhitov R (2003). Toll pathway-dependent blockade of CD4+CD25+ T cell-mediated suppression by dendritic cells. Science 299: 1033-1036.http://dx.doi.org/10.1126/science.1078231

Phan GQ, Yang JC, Sherry RM, Hwu P, et al. (2003). Cancer regression and autoimmunity induced by cytotoxic T lymphocyte-associated antigen 4 blockade in patients with metastatic melanoma. Proc. Natl. Acad. Sci. USA 100: 8372-8377.http://dx.doi.org/10.1073/pnas.1533209100

Rathore AS, Kumar S, Konwar R, Makker A, et al. (2014). CD3+, CD4+ \& CD8+ tumour infiltrating lymphocytes (TILs) are predictors of favourable survival outcome in infiltrating ductal carcinoma of breast. Indian J. Med. Res. 140: 361-369.

Rivera MP, Detterbeck F and Mehta AC; American College of Chest Physicians (2003). Diagnosis of lung cancer: the guidelines. Chest 123 (Suppl): 129S-136S. http://dx.doi.org/10.1378/chest.123.1_suppl.129S

Robinson LA, Ruckdeschel JC, Wagner H, Jr., Stevens CW, et al. (2007). Treatment of non-small cell lung cancer-stage IIIA: ACCP evidence-based clinical practice guidelines (2nd edition). Chest 132: 243S-265S.

Rosenberg SA, Yang JC and Restifo NP (2004). Cancer immunotherapy: moving beyond current vaccines. Nat. Med. 10: 909-915.http://dx.doi.org/10.1038/nm1100

Schaefer C, Kim GG, Albers A, Hoermann K, et al. (2005). Characteristics of CD4+CD25+ regulatory T cells in the peripheral circulation of patients with head and neck cancer. Br. J. Cancer 92: 913-920. http://dx.doi.org/10.1038/ sj.bjc. 6602407

Schoo WH and Coppes L (1976). Use of palatal mucosa and lyophilized dura mater to create attached gingiva. J. Clin. Periodontol. 3: 166-172.http://dx.doi.org/10.1111/j.1600-051X.1976.tb01864.x

Scott WJ, Howington J, Feigenberg S, Movsas B, et al. (2007). Treatment of non-small cell lung cancer stage I and stage II: ACCP evidence-based clinical practice guidelines (2nd edition). Chest 132: 234S-242S.

Yu DP, Han Y, Zhao QY and Liu ZD (2014). CD3+ CD4+ and CD3+ CD8+ lymphocyte subgroups and their surface receptors NKG2D and NKG2A in patients with non-small cell lung cancer. Asian Pac. J. Cancer Prev. 15: 26852688. http://dx.doi.org/10.7314/APJCP.2014.15.6.2685 